ScIDice

\section{Central Odontogenic Fibroma Associated with Eruption Disturbance of a Permanent Incisor in a Six-Year-Old Girl}

Case Report

\author{
Nakamura $\mathrm{Y}^{1^{*}}$, Masuda $\mathrm{K}^{2}$, Yamaza $\mathrm{H}^{3}$, Nonaka $\mathrm{K}^{4}$ \\ ${ }^{1}$ Assistant Professor, Department of Oral Biological Science, Niigata University Graduate School of Medical and Dental Sciences, Gakkocho-dori, \\ Chuo-ku, Niigata, Japan. \\ ${ }^{2}$ Lecturer, Department of Pediatric Dentistry and Special Needs Dentistry, Kyushu University Hospital, Maidashi, Higashi-ku, Fukuoka, Japan. \\ ${ }^{3}$ Lecturer, Department of Dental Science, Kyushu University, Maidashi, Higashi-ku, Fukuoka, Japan. \\ ${ }^{4}$ Professor, Department of Dental Science, Kyushu University, Maidashi, Higashi-ku, Fukuoka, Japan.
}

\title{
Abstract
}

Dentists occasionally encounter unilocular radiolucent lesions that may be associated with a wide spectrum of conditions in the jaw. Central odontogenic fibroma (COF) is a rare fibroblastic neoplasm of the jaw, which presents as a wellcircumscribed unilocular radiolucent lesion often associated with an impacted tooth. Histologically, COF is composed of collagenous fibrous connective tissue containing varying amounts of inactive odontogenic epithelium. Based on the clinical, radiological and histological characteristics, COF is not easily differentiated from an enlarged dental follicle, or a fibromatous or cystic lesion of jaw. We describe a case of a 6-year-old girl with a unilocular radiolucent lesion around the crown of the impacted right permanent central incisor in the maxilla. In addition to clinical and radiological examination, an incisional biopsy was performed to make a diagnosis of COF and to determine the best treatment, which included a surgical procedure and postoperative occlusal guidance. The patient was followed up for 8 years and developed a satisfactory anterior occlusion with no signs of recurrence of the COF. Differential diagnosis and surgical procedures for COF associated with tooth eruption in a young child are discussed.

\section{Introduction}

Central odontogenic fibroma $(\mathrm{COF})$ is a rare fibroblastic neoplasm in the jaw, characterized by varying amounts of inactivelooking odontogenic epithelium embedded in a mature fibrous stroma [1-3]. Radiographically, it usually appears as a unilocular radiolucency with well-defined borders, and is often associated with an unerupted or displaced tooth. It is often confused with dental follicles of developing tooth germs or cystic lesions of the jaw [4-9], because periapical inflammation of the necrotic pulp of deciduous teeth occasionally appears to spread over the dental follicle of developing permanent successors. Therefore, comprehensive assessment of clinical, radiological and histological findings is particularly important in a radiolucent lesion associated with a tooth germ in a child to make a proper diagnosis and choose the best treatment.
This case report describes a COF found in the anterior maxilla of a child with a mixed dentition. The lesion involved both the root of a non-vital deciduous tooth and the crown of an unerupted permanent successor, and resembled an inflammatory maxillary cyst commonly observed during the mixed dentition period [10]. An incisional biopsy, in combination with clinical and radiological examinations, was helpful in making a diagnosis of COF. This was followed by surgical treatment and postoperative eruption guidance.

\section{Case Report}

A 6-year-old Japanese girl was referred to our hospital by a general dentist who suspected a developmental anomaly of the tooth germs of the permanent incisors in the right maxilla. She had no systemic disorders, no problems of growth and development, and

Yuki Nakamura,

Assistant Professor, Department of Oral Biological Science, Niigata University Graduate School of Medical and Dental Sciences, Japan.

Tel: $+81-25-227-2999$

Fax: +81-25-227-2998

Email: nakayuki@dent.niigata-u.ac.jp

Received: February 11, 2016

Accepted: March 03, 2016

Published: March 08, 2016

Citation: Nakamura Y, Masuda K, Yamaza H, Nonaka K (2016) Central Odontogenic Fibroma Associated with Eruption Disturbance of a Permanent Incisor in a Six-Year-Old Girl. Int J Dentistry Oral Sci. 03(3), 205-208. doi: http://dx.doi.org/10.19070/2377-8075-1600043

Copyright: Nakamura $\mathbf{Y}^{\circ}$ 2016. This is an open-access article distributed under the terms of the Creative Commons Attribution License, which permits unrestricted use, distribution and reproduction in any medium, provided the original author and source are credited. 
no history of dental trauma. The intraoral examination revealed fused right maxillary deciduous incisors with a resin restoration (Figure 1). There was no pain, swelling, redness or other inflammatory signs in the gingiva in this region. In the left maxilla, a permanent central incisor had started erupting and a deciduous lateral incisor was still present.

Panoramic and periapical radiographs showed a small radiolucency associated with the partially resorbed roots of the fused deciduous incisors (Figure 2). The unerupted maxillary right permanent central incisor was suspected to be involved in the lesion. Radiopaque material was found in the pulp of the fused deciduous incisors, indicating previous endodontic therapy. The tooth germ of the maxillary right permanent lateral incisor was missing. A computed tomograph revealed that a small and well-defined radiolucent lesion was associated with the root of the fused deciduous incisors as well as the crown of the unerupted permanent central incisor displaced in a labial ectopic position (Figure 3). Based on these findings, a clinical diagnosis of maxillary cyst or neoplasm involving the unerupted maxillary permanent central incisor was made. Eruption of the maxillary right permanent central incisor was thought to have been interfered with by the radiolucent lesion around the root of the fused deciduous incisors. The maxillary right permanent lateral incisor was suspected to be congenitally missing.

For a differential diagnosis, an incisional biopsy was performed in combination with extraction of the fused maxillary right deciduous incisors. Under local anesthesia, the deciduous incisors were extracted followed by excision of pathologic tissue to partially expose the crown of the unerupted maxillary right permanent central incisor (Figure 4). The lesion inside was solid. Microscopic examination of the excised tissue showed dense fibrous connective tissue including islets and strands of odontogenic epithelium (Figure 5). The odontogenic epithelium did not include a peripheral layer of cuboidal or columnar cells, or stellate reticulum. There was no surface epithelial layer, myxoid change, or calcific deposits. Based on these histopathological findings, a diagnosis of COF was established.

COF has limited growth potential, and the prognosis is good after enucleation with bone curettage [1-3]. In the present case, the maxillary right permanent central incisor was affected and the ipsilateral permanent lateral incisor was missing. Therefore, we decided to preserve the affected permanent central incisor for physiological and esthetic development of the anterior occlusion. Under general anesthesia, the residual pathological tissue was enucleated and the bone was curetted as completely as possible without injuring the affected permanent central incisor. In addition, an orthodontic button was attached to the exposed lingual enamel of the right permanent central incisor for postoperative traction. Microscopic examination of the residual lesion confirmed the diagnosis of COF. One month after the surgery, a device for orthodontic traction was applied to the affected permanent central incisor (Figure 6). Two months later, the central incisor began to erupt. The tooth was guided towards the occlusal plane and erupted into a normal position 4 months after the beginning of traction. The device was then replaced by a lingual arch as a space

Figure 1. Intraoral photograph at the initial visit.

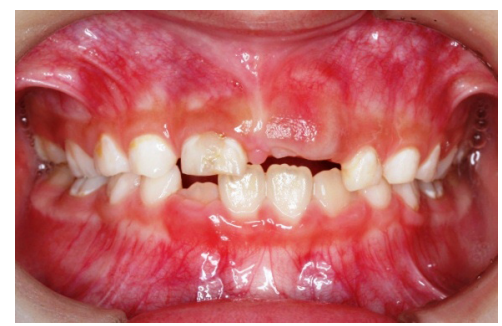

Figure 2. Periapical radiograph of the anterior maxilla and panoramic radiograph at the initial visit.
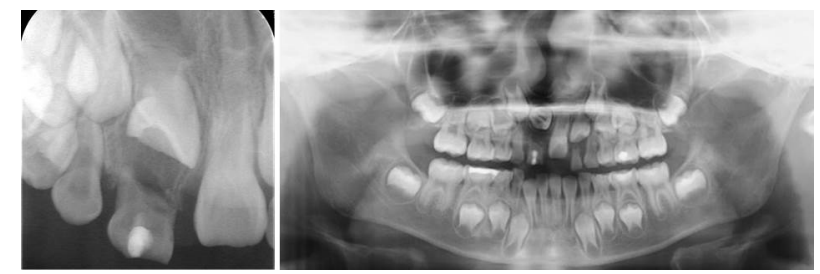

Figure 3. Computed tomographic images of the lesion; sagittal (left) and frontal (right) views.
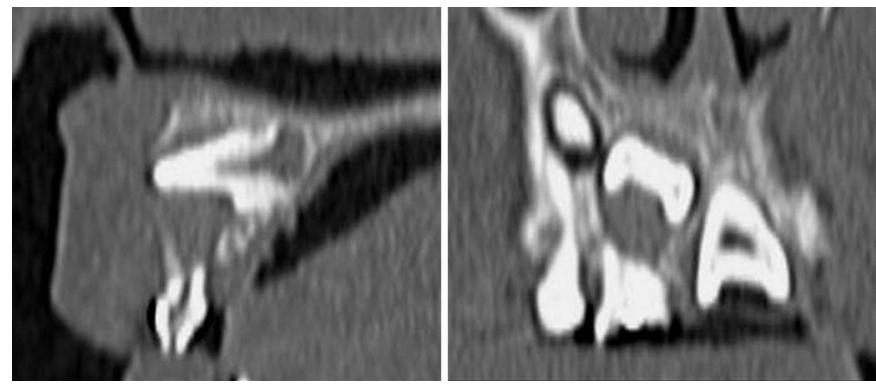
Figure 4. Intraoral photograph during incisional biopsy (left); the excised sample (right).

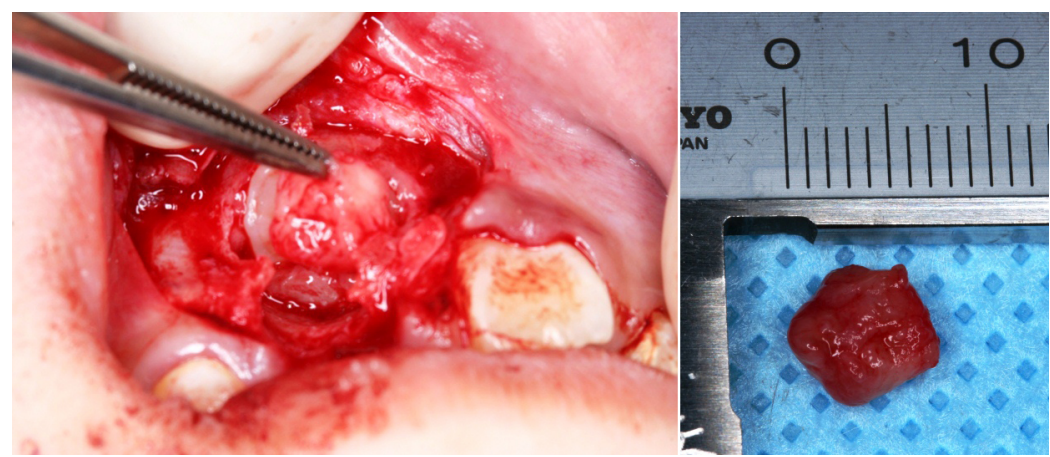

Figure 5. Low (40X) and high (100X) magnification showing the histopathological appearance of the biopsy sample (hematoxylin and eosin staining).

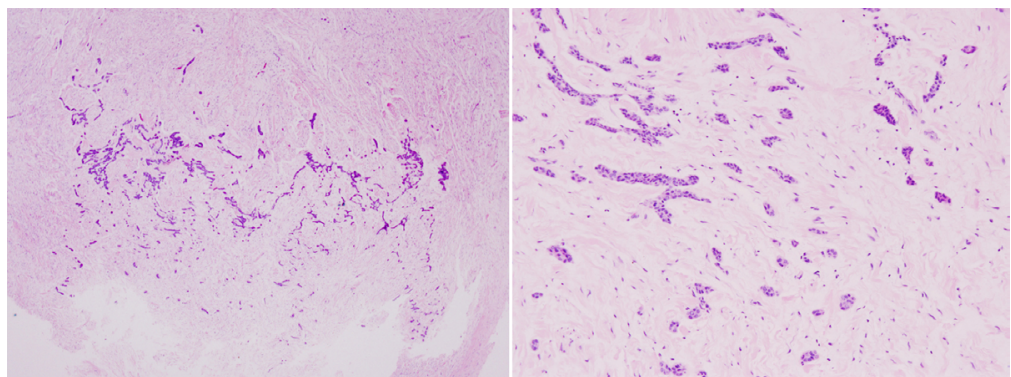

Figure 6. Intraoral appearance after the surgery. At the beginning of postoperative traction (A). After 2 months, the affected permanent central incisor has begun to erupt (B). After 4 months of traction (C).
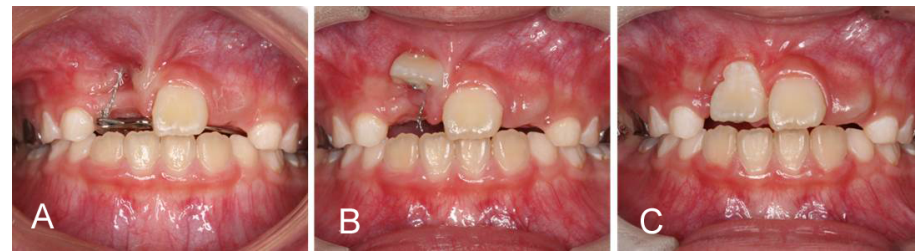

retainer. The postoperative course was uneventful (Figure 7). The patient was followed up for 8 years, and developed an acceptable anterior occlusion with no signs of recurrence.

\section{Discussion}

In children with mixed dentition, unilocular and well-defined radiolucent lesions occasionally occur around deciduous teeth as well as permanent tooth germs. Although a wide spectrum of conditions exhibit these radiographic features, benign odontogenic lesions, such as radicular cysts or dentigerous cysts, are more frequent $[11,12]$. Many can be diagnosed based on the clinical and radiographic findings, and conservative treatment, such as marsupialization or simple enucleation, leads to a satisfactory outcome. However, all excised tissues should be submitted for histopathologic examination to rule out tumors or invasive lesions [13].

$\mathrm{COF}$ is a rare benign fibroblastic neoplasm of the jaw [1-3]. Clinically, it tends to expand slowly and manifest as a painless cortical expansion, which is sometimes associated with displacement, eruption disturbance or root resorption of the adjacent teeth. In half of cases the tumor appears as a unilocular radiolucent area with well-circumscribed margins, although it may occasionally appear as a multilocular or mixed radiolucent and radiopaque area
$[2,3]$. Histopathologic examination should be a component of a proper evaluation of the lesion, because differentiating $\mathrm{COF}$ from other lesions in the jaw can be difficult, clinically and radiographically. In this case, a well-defined unilocular radiolucent lesion involved both the partially resorbed root of a non-vital deciduous tooth and the crown of an unerupted and dislocated permanent successor. The lesion was small, and the swelling of the affected area was not conspicuous, although the cortical bone was slightly expanded. These clinical and radiographic findings were most suggestive of a cystic lesion [10-12]. However, tissue obtained at biopsy revealed the histopathologic picture of COF.

Histopathologically, COF is composed of mature collagen fibers with numerous interspersed fibroblasts [1-3]. It contains a varying amount of inactive-looking odontogenic epithelium resembling dental lamina. According to the amount of odontogenic epithelium, there are two histopathologic variants of COF: epithelial poor and epithelial rich [3]. Epithelial poor COF appears as a non-invasive connective tissue lesion with scattered remnants of inactive-looking odontogenic epithelium. Delicate acellular fibers are interspersed in the ground substrate with occasional fibromyxoid change. Thus, this variant resembles a dental follicle histopathologically. Epithelial rich COF is composed of cellular fibroblastic connective tissue containing islands or strands of 
Figure 7. Intraoral appearance and periapical radiograph 5 years after the surgery.
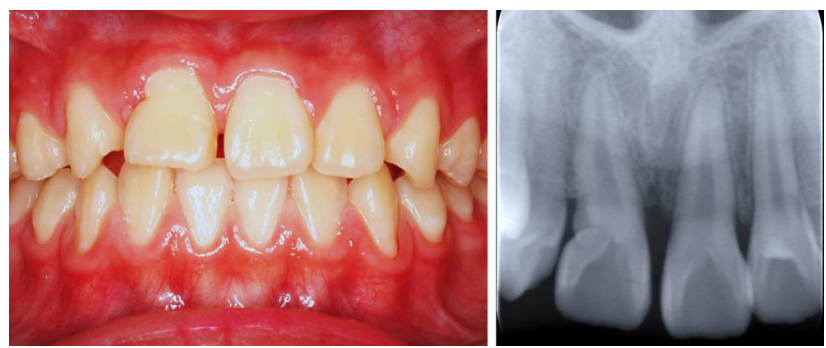

inactive-looking odontogenic epithelium that are integral components of this variant. In the current case, the lesion was composed of relatively dense fibrous tissue containing inactive-like odontogenic epithelial islands without any myxoid change or epithelial lining. These findings were most suggestive of epithelial rich $\mathrm{COF}$, rather than other fibromatous conditions, such as ameloblastic fibroma, odontogenic myxoma, desmoplastic fibroma, or dental follicle [1, 12-14]. In particular, odontogenic myxoma and desmoplastic fibroma need to be considered in the differential diagnosis because they exhibit aggressive and infiltrative behavior with a much higher recurrence rate than $\mathrm{COF}[15,16]$.

General treatment of COF is enucleation with bone curettage because of the lack of aggressive features and the rarity of recurrence [1-3]. An embedded tooth is preferably extracted with the lesion to avoid incomplete surgical removal and to minimize the risk of recurrence. In the case presented here, two major factors were considered before the surgery. Firstly, the lesion was small and localized around the crown of the unerupted permanent central incisor. Secondly, the ipsilateral permanent lateral incisor was congenitally missing, possibly associated with the fused deciduous predecessor. Therefore, we decided to preserve the affected permanent central incisor in order to guide the anterior occlusion physiologically and esthetically. Enucleation and bone curettage were carefully performed as completely as possible, while minimizing injury to the affected permanent central incisor. However, conservative treatment may result in incomplete removal of the lesion, making the risk of recurrence higher [17]. Careful and long-term follow-up is required for early recognition of recurrence.

\section{Conclusion}

In pediatric patients, cystic radiolucent lesions around developing tooth germs with or without eruption disturbance are sometimes observed. These lesions include a wide spectrum of conditions ranging from benign cysts to aggressive tumors. Because of the various sizes of the lesions with or without affected teeth, diagnosis can be problematic. Comprehensive assessment of clinical, radiographic and histopathologic findings of the lesion is strongly recommended to avoid misdiagnosis leading to under treatment or over treatment.

\section{References}

[1]. Gardner DG (1996) Central odontogenic fibroma current concepts. J Oral Pathol Med 25(10): 556-561.

[2]. Reichart PA, Philipsen HP (2004) Odontogenic tumors and allied lesions. Quintessence Publishing Co, Ltd, Illinois.

[3]. Barnes L, Eveson JW, Reichart P, Sidransky D (2005) World Health Organization Classification of Tumors: Pathology and genetics of head and neck tumors. IARC Press, Lyon, New York.

[4]. Lukinmaa PL, Hietanen J, Anttinen J, Ahonen P (1990) Contiguous enlarged dental follicles with histologic features resembling the WHO type of odontogenic fibroma. Oral Surg Oral Med Oral Pathol 70(3): 313-317.

[5]. Kim J, Ellis GL (1993) Dental follicular tissue: misinterpretation as odontogenic tumors. J Oral Maxillofac Surg 51(7): 762-768.

[6]. Hirschberg A, Buchner A, Dayan D (1996) The central odontogenic fibroma and the hyperplastic dental follicle: study with Picrosirius red and polarizing microscopy. J Oral Pathol Med 25(3): 125-127.

[7]. Daniels JS (2004) Central odontogenic fibroma of mandible: a case report and review of the literature. Oral Surg Oral Med Oral Pathol Oral Radiol Endod 98(3): 295-300.

[8]. Ikeshima A, Utsunomiya T (2005) Case report of intra-osseous fibroma: a study on odontogenic and desmoplastic fibromas with a review of the literature. J Oral Sci 47(3): 149-157.

[9]. Barnes L, Chiosea SI, Seethala RR (2010) Head and neck Pathology. Demos Medical Publishing, New York.

[10]. Benn A, Altini M (1996) Dentigerous cysts of inflammatory origin. A clinicopathologic study. Oral Surg Oral Med Oral Pathol Oral Radiol Endod 81(2): 203-209.

[11]. Bodner L (2002) Cystic lesions of the jaws in children. Int J Pediatr Otorhinolaryngol 62(1): 25-29.

[12]. Schmitd LB, Bravo-Calderón DM, Soares CT, Oliveira DT (2014) Hyperplastic dental follicle: a case report and literature review. Case Rep Dent 2014: 251892.

[13]. Stoelinga PJ (2012) The management of aggressive cysts of the jaws. J Maxillofac Oral Surg 11(1): 2-12.

[14]. Regezi JA (2002) Odontogenic cysts, odontogenic tumors, fibroosseous, and giant cell lesions of the jaws. Mod Pathol 15(3): 331-341.

[15]. Harokopakis-Hajishengallis E, Tiwana P (2007) Odontogenic myxoma in the pediatric patient: a literature review and case report. Pediatr Dent 29(5): 409-414.

[16]. Said-Al-Naief N, Fernandes R, Louis P, Bell W, Siegal GP (2006) Desmoplastic fibroma of the jaw: a case report and review of literature. Oral Surg Oral Med Oral Pathol Oral Radiol Endod 101(1): 82-94.

[17]. Melo AR, Santos Tde S, do Amaral MF, Albuquerque Dde P, Andrade ES, et al. (2011) Recurrence of central odontogenic fibroma: a rare case. Gen Dent 59(2): e78-81. 